\title{
EXAMINATION OF REYNOLDS NUMBER EFFECT ON THE DEVELOPMENT OF ROUND JET FLOW
}

\author{
Olanrewaju Miracle Oyewola $\bowtie$ \\ School of Mechanical Engineering \\ Fiji National University \\ Ratu Mara Road, Samabula, Suva, Fiji, P.O. Box 3722 \\ Department of Mechanical Engineering ${ }^{l}$ \\ oooyewola001@gmail.com,olanrewaju.oyewola@fnu.ac.fj \\ Adebunmi Okediji \\ Department of Mechanical Engineering ${ }^{I}$ \\ Department of Mechanical Engineering \\ Elizade University \\ P.M.B. 002, Ilaramokin, Ondo State, Nigeria, 340271 \\ Olusegun Olufemi Ajide \\ Department of Mechanical Engineering ${ }^{l}$ \\ Muyiwa Samuel Adaramola \\ Faculty of Environmental Sciences and Natural Resource Management \\ Norwegian University of Life Sciences \\ 1430 Elizabeth Stephansens vei 15, As, Norway, P.O. Box 5003 \\ ${ }^{1}$ University of Ibadan \\ Sango-Ojo road, Ibadan, Oyo State, Nigeria, 200284
}

$\triangle$ Corresponding author

Abstract

In this study, the Reynolds number effect on the development of round jet flow is presented. The jet is produced from a smoothly contracting round nozzle and the flow structure is controlled by varying the air blower speed in order to obtain various Reynolds numbers (Re). The flow Reynolds number considered varies between 1140 and 9117. Mean velocity measurements were taken using hot-wire probe at different axial and lateral distances $(0 \leq x / d \leq 50$, where $x$ is the downstream distance and $d$ is the nozzle diameter) for the jet flow and at for $0 \leq x / d \leq 30$ in long pipe attached to the nozzle. Measurements reveal that Reynolds number dictate the potential core length such that the higher the Reynolds number, the lower the potential core which is a measure of mixing of jet and ambient fluid. It shows that further away from the jet exit section, potential core decreases as Reynolds number increases, the velocity profile has a top hat shape very close to the nozzle exit and the shape is independent of Reynolds number. It is found that potential core extends up to $x / d=8$ for Reynolds number of 1140 as against conventional near field $0 \leq x / d \leq 6$. This may suggest effect of very low Reynolds number. However, further investigation is required to ascertain this at extremely low Reynolds numbers. It is also observed that further away from the jet exit section, the higher the downstream distance, the higher the jet half-width $\left(R_{1 / 2}\right)$. Furthermore, the flow in the pipe shows almost constant value of normalised axial centerline velocity for a longer distance and this clearly indicates that there is mass redistribution rather than entrainment of ambient fluid. Overall, the Reynolds number controls the magnitude rather than the wavelength of the oscillation.

Keywords: Centerline velocity, Jet half-width, Potential core, Reynolds number, Round jet.

DOI: $10.21303 / 2461-4262.2021 .001872$

\section{Introduction}

Due to various applications of jet flows such as in combustion processes, chemical processes, pollutant discharge, cooling, mixing and drying processes, many researchers have studied the structure and development of jets in order to achieve certain target [1]. Depending on the sources of the flow, jet flow can take different shape. However, the most study type of jet flow is the one that originated from round shape source, which is generally referred to as 'round jet flow'. This is 
due to easy set-up in experimental and numerical studies as well as its wide range of applications. Recent research on this subject has resulted in much improved understanding on the process by which the jet evolves, its stability and how the surrounding fluid is entrained into the jet flow at the outlet of the jet. Three different regions have been defined in the axisymmetric round jet flow. They are the near field, the intermediate field and the far field regions [2]. The near field region (often referred to the region that contains the potential core) where the flow characteristics match those of the nozzle exit and is usually within $0 \leq x / d \leq 6$ (where $x$ is downstream distance and $d$ is nozzle diameter). The far field is located at approximately $x / d \geq 30$ where it is classified as the self-similar region. The intermediate-field region lies between the near and far field, which is $6<x / d<30$. The near and intermediate fields together comprise the developing portion of the jet.

There are three basic types of circular jet nozzle; a smooth contraction, a long pipe and a sharp-edge orifice plate. For detailed review of jet flow, see [3-5]. Considerable work has been documented on round jets. For instance, [6] investigated potential core length of round jets in stagnant and moving environments such as co-flow and counter flow using particle image velocimetry at Reynolds number of 4000 . They discovered that decay constant (which is defined as rate at which jet deviates from centerline along downstream) increased in co-flow than in counter flow and that moving fluid reduces the potential core length [7] experimentally considered the behaviour of the round jet for Reynolds numbers of 4000, 6000 and 8000 measured within $0 \leq x / d \leq 10$ using hotwire anemometer. They reported that mean velocity showed negligible difference in the near field. Furthermore, away from near field, [8] considered Reynolds number between 1000 and 5000 and measured velocity and concentration using Laser Doppler anemometry within 40-jet exit diameter. They reported that as Reynolds number increases, potential core decreases. Similarly, [2] considered Reynolds number between 6.000 and 100,000 showed that there is decreased in magnitude of the length scale as Reynolds number increases and weakly dependent on radial position. However, they found that beyond potential core, laminar length scale to viscous length scale exceeds unity [1] carried out measurements at Reynolds numbers of 6000, 10,000 and 30,000 and observed that turbulence intensity is anisotropy and axial turbulence intensity is higher than the lateral turbulent intensity. It should be noted that their measurements were within $0 \leq x / d \leq 25$. In the far field, [9] investigated effect of initial conditions on the far field of a round jet using hot-wire anemometer, they discovered that second order velocity profile is not affected by change in Reynolds number and that coherent structure evolves rapidly to equilibrium. [10] studied the influence of initial conditions on the evolution towards similarity in turbulent round jets by considering two jets with diameters $D=63.5 \mathrm{~mm}$ and $D=47.8 \mathrm{~mm}$. They found that velocity decrease faster in larger jet.

Interestingly, attempt has been made on fully developed jet flow in a long pipe. For instance, [11] studied secondary structures in developed turbulent jet for Reynolds number between 3000 and 30,000. They found that vortical structures size in transverse planes decreases as Reynolds number increases and vortex ring was lost away from near field and in the far field. In addition, energy increased from primary to secondary vortex formation resulting in higher radial distances [12] studied flow in axisymmetric pipe jet for Reynolds number of 5500, different shear layer was obtained compared with contraction jet and mean velocity was first obtained in selfsimilar region followed by fluctuation aspect.

Despite the numerous works that has been done on jet flow, there is paucity of information on the structure of flow in the case of long pipe circular nozzle especially at various Reynolds numbers. Fundamentally, this information will improve our understanding and create a platform for better control strategies. Also, there is need to shed more light on the region of potential core over a range of Reynolds numbers. This will be valuable especially in the application relating to pollutant discharge.

In this study, the aim is to experimentally examine how Reynolds number influence the development of round jet flow for Reynolds number range between 1140 and 9117 which covers the low, intermediate and high Reynolds number regimes. The objectives are to examine the influence of Reynolds number on the following: mean centerline velocity, spread of mean velocity, velocity profiles and centerline velocity of confined jet as compared with free jet of the same Reynolds number. This is necessary in order to improve our understanding of the flow development in free jet as well as its behaviour in the attached pipe over various flow regimes. 


\section{Materials and methods}

Fig. 1 shows the schematic arrangement of the apparatus used for this study. It consists of air blower $(1.5 \mathrm{~kW})$, a settling chamber and a smoothly contracting round nozzle (contraction ratio 12:1), which discharge air into a laboratory room. A flow straightening section made of drinking straws (each straw is $100 \mathrm{~mm}$ long and $4 \mathrm{~mm}$ inner diameter) were placed inside the chamber to reduce the turbulence level in the incoming flow. Variable transformer was also used to control the flow from the blower in order to achieve the desired velocity.

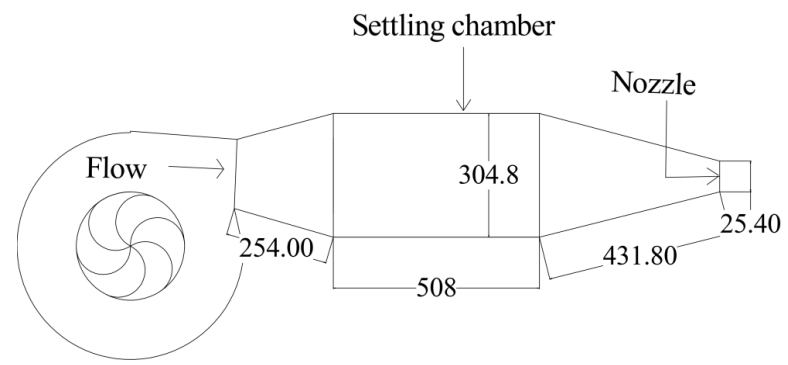

Fig. 1. Descriptive layout of the apparatus (all dimensions in mm, not drawn to scale)

Six different Reynolds numbers $\left(\operatorname{Re}=U_{j} d / \mathrm{v}\right.$, where $U_{j}=$ jet exit velocity, $d=$ nozzle diameter and $v$ is the kinematic viscosity of air) based on nozzle exit diameter of $25.4 \mathrm{~mm}$ were considered (1140, 1465, 1791, 5210, 7000 and 9117).

Single hot-wire anemometer (velocity range $0.2-17.0 \mathrm{~m} / \mathrm{s}$ ) with its probe $(5 \mu \mathrm{m}$ tungsten, $0.94 \mathrm{~m}$ ) was used to measure mean stream-wise and lateral velocities at various distances from the nozzle exit within $0 \leq x / d \leq 50$. In addition, the centerline velocity measurement was taken in long steel pipe within $0 \leq x / d \leq 30$ for Reynolds numbers 5210,7000 and 9117 . The velocity at the nozzle exit was constantly checked to ascertain the correctness of the measurements. Using propagation of error analysis, the uncertainty in the measurement of velocity was about $\pm 5 \%$. This was estimated by measuring velocity 5 times at several locations relative to the nozzle exit and at each location, the uncertainty was $\pm 5 \%$ of the mean value.

\section{Results and Discussion}

Fig. 2 shows the inverse mean centerline velocity. Also included for comparison are the results of $[1,7]$.

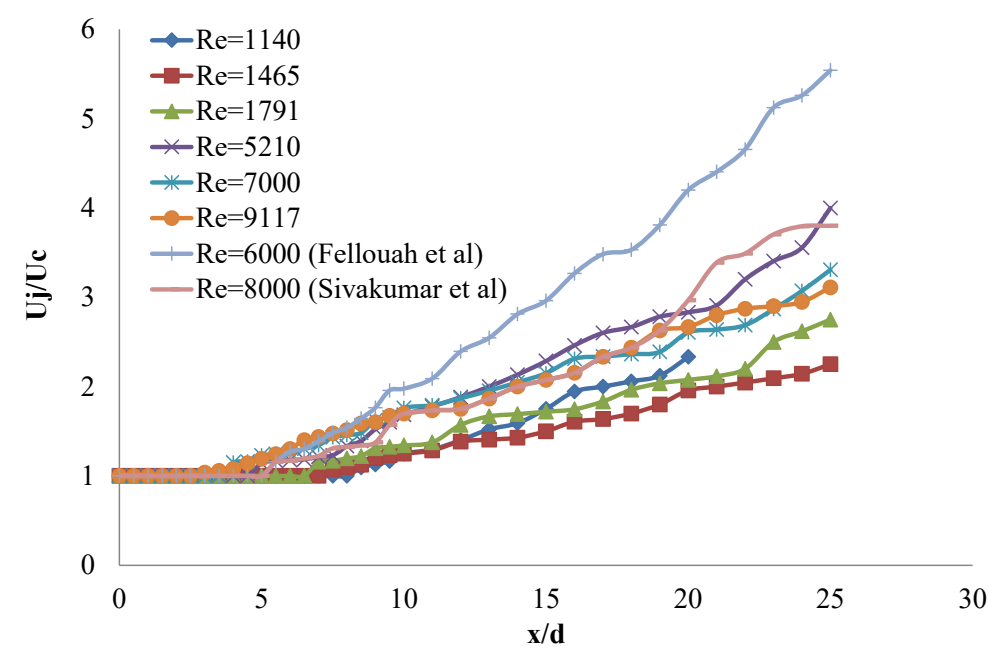

Fig. 2. Axial decay of centerline mean velocity (Developing region)

The mean flow spreads out gradually and axis-symmetrically and reveals a 'potential core', where the mean velocity is approximately uniform. For the Reynolds number considered (from 
1140 to 9117), potential core occurs at different downstream position. At the Reynolds number (Re) of 1140 , potential core is $8 \mathrm{~d}$ from the nozzle exit (i.e. $x / d=8$ ), while for other Reynolds numbers $1465,1791,5210,7000$ and 9117 , the potential cores were $7 \mathrm{~d}, 6.5 \mathrm{~d}, 4.5 \mathrm{~d}, 3.5 \mathrm{~d}$ and $2.5 \mathrm{~d}$, respectively.

The distribution shows that as Reynolds number increase, the potential core decrease. The results suggest that mixing of fluids is enhanced at high Reynolds number. This is not surprising since the interaction among various flow structures is increased and this thereby leads to higher momentum transfer. The Reynolds number of 9117 has the lowest potential core and therefore, it is considered as best for the mixing processes. For comparison with previous measurements in smoothly contracting nozzle, the data by [1], $R e=6000$ and that of [7], $R e=8000$ were included in Fig. 2 and good agreement is observed in the potential core. It should be noted at $R e=1140$, the potential core is $8 \mathrm{~d}$ which is outside the suggested range $(0 \leq x / d \leq 6)$. This may reflect the effect of extremely low Reynolds number on the shear layer. However, it is not surprising since mixing at this Reynolds number may not be strong and this may likely alter the potential core. Further investigation at lower Reynolds numbers to substantiate this is required.

Furthermore, away from the end of potential core, it is interesting to note that centerline velocity begins to decrease (note that the normalisation method shows upward trend). Fig. 2 shows the decrease up to $x / d=25$ which is referred to as developing region. The distributions clearly show the effect of entrainment of ambient fluid (air) into the jet. In order to conserve the momentum, as the centerline velocity decrease with downstream distance, the mass flow of the jet progressively increase as a result of ambient fluid entrainment and this also respond to change in Reynolds number. For instance, at $x / d=10$, for $R e=1140$, the normalised velocity is 1.4 , while it is 1.6 and 1.75 for $R e=1791$ and $R e=9117$, respectively. Similarly, at $x / d=15$, for $R e=1140$, the normalised centerline velocity is 1.75 , while 1.83 and 2.07 for $R e=1791$ and $R e=9117$, respectively. The distributions are similar in the downstream region up to $x / d=25$. This clearly shows that as Reynolds number is increased, centerline velocity also increases in the intermediate region. Although, [1] stated that there is no significant variation in the centerline velocity beyond the potential core maybe because of the higher Reynolds numbers considered in their work $(R e=6000-30,000)$. Conversely, [7] argued that variations of centerline velocities had slight deviations in the near field region, which possibly may be due to amount of flow entrainment near jet exit as reported by $[13,14]$. In this study, centerline velocity responds to change in Reynolds number, clusters in data are possibly due to entrainment of ambient air. Therefore, the distributions of centerline velocities are the same but there is variation in magnitude in response to Reynolds number with higher values recorded with increasing Reynolds number.

Fig. 3 shows decay of centerline velocity in the far field. In this region, the distribution of centerline velocity clearly shows dependence on Reynolds number. It is interesting to note that lower Reynolds numbers 1140, 1465 and 1791 decay at $x / d=25$. However, Reynolds numbers 5210, 7000 and 9117 were considered in the far field. For the range of Reynolds numbers considered in this study, as downstream distance increase, there is corresponding decrease in centerline velocity except at $x / d=40$ where centerline velocity of Reynolds number $R e=9117$ crosses that of $R e=7000$, this occurs possibly as a result of ambient fluid entrainment. The work of [9] was used for comparison and the same trend was observed.

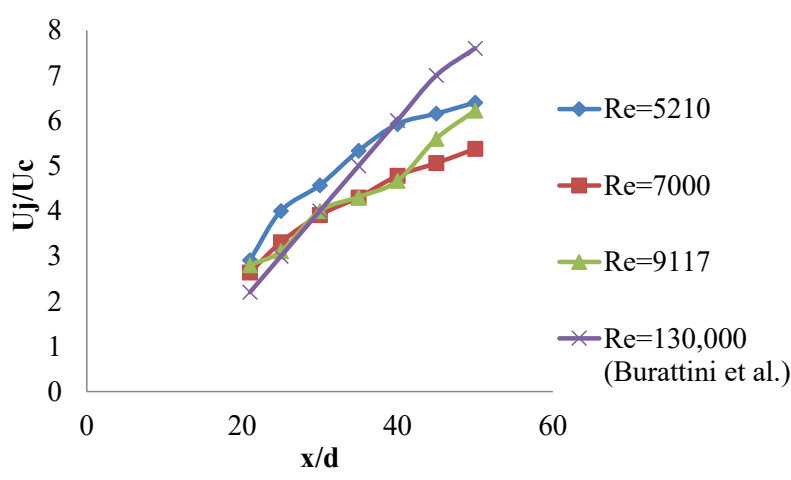

Fig. 3. Axial decay of centerline mean velocity (far field) 
The decay constant and virtual origin of the jet flow was estimated using (1), [1]:

$$
\frac{U_{j}}{U_{c}}=\frac{1}{B}\left(\frac{x}{d}-\frac{x_{o}}{d}\right),
$$

where $x_{o}$ is the virtual origin and $B$ is the decay constant, $\mathrm{x}$ is the downstream distance, $d$ is the nozzle diameter, $U_{c}$ is the centerline velocity and $U_{j}$ is the jet exit velocity. In this study, the decay constant is presented in Table 1 and those obtained from the literature. Good agreement is found between the present decay constant and those found in the literature. The decay constant seems to be slightly independent of the Reynolds number but appears to depend on the region of measurement. However, the effect of low Reynolds numbers cannot be completely ruled out. In general, the centerline velocity decays faster in the intermediate region than in the self-similar region due to the flow development.

Table 1

Decay constant of mean centerline velocity

\begin{tabular}{|c|c|c|c|c|}
\hline Authors & Reynolds number & $x / d$ & $x_{o} / d$ & $B$ \\
\hline Present study & 5,000 & $7.5-25$ & 2.7 & 5.92 \\
\hline Xu and Antonia (2002) [14] & 86,000 & $20-75$ & 3.7 & 5.6 \\
\hline Fellouah et al. (2009) [1] & 10,000 & $15-29$ & 2.5 & 5.59 \\
\hline Ferdman et al. (2000) [15] & 24,000 & $\geq 15$ & 2.5 & 6.7 \\
\hline Sivakumar et al. (2012) [7] & 6,000 & $<15$ & 2.87 & 4.83 \\
\hline
\end{tabular}

While the previous results indicated some Reynolds number dependence, the normalised jet half-width should further reinforce these changes. Fig. 4 shows the spread of mean velocity in both near and far field regions $(0 \leq x / d \leq 50)$, a normalised jet half-width $R_{1 / 2}$ (point in the radial direction where velocity is equal to half of the centerline velocity) plotted against a normalised downstream distance. As expected, the higher the downstream distance, the higher the jet halfwidth. Interestingly, it seems to depend on Reynolds number and entrainment of the ambient air. For instance, at a distance $x / d=5$, the jet half-width is 0.7 for $R e=5210$ while it is 0.72 for $R e=7000$ and 0.75 for $R e=9117$. Therefore, the higher the Reynolds number, the higher the spread rate of the mean velocity. Little significance is observed in near field unlike far field where significant change is observed. Jet half-width decays at $x / d=25$ for the lower Reynolds number (i.e. $R e=1140-1791$ ) whereas it extends to $x / d=50$ for the higher Reynolds number considered, this further establish that the higher the Reynolds number, the further the effect in the downstream of the jet. In the far field, Reynolds number controls the response of the jet spread. For instance, at the far end (i.e $x / d=50$ ), jet half-with is 6.1 for $R e=9117$ whereas it is 5.25 and 4.24 for $R e=7000$ and 5210 , respectively.

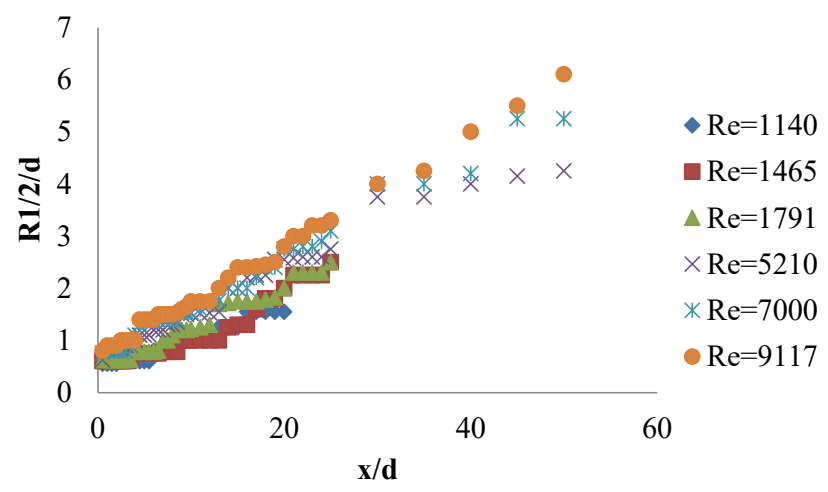

Fig. 4. Spread rate of mean velocity (Near to far field) 
Fig. 5 shows the streamwise velocity profiles at $x / d=0.5$. This constitutes the streamwise velocity profile in the near field region of the flow. The velocity profile is one of the initial conditions used in jet flow to characterise the flow field. It can be observed from Fig. 6 that the profile for the round jet has a top-hat shape (a shape in which axisymmetric jet has lateral velocity that decrease uniformly away from the centre). The profile is independent of Reynolds number and hence, the similar profile is obtained. For the Reynolds numbers considered in this study, the peak-normalised velocities occur at the centerline and decrease laterally due to the entrainment of ambient air. However, it is almost the same for all the Reynolds numbers. It can be deduced that at the nozzle exit section, irrespective of Reynolds numbers, the profile is the same with a minimal difference in magnitude of the normalised velocity.

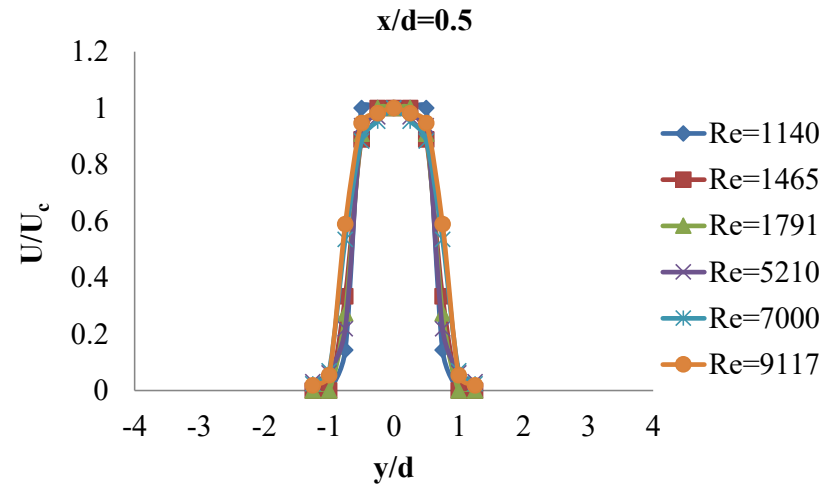

Fig. 5. Streamwise mean velocity at $x / d=0.5$

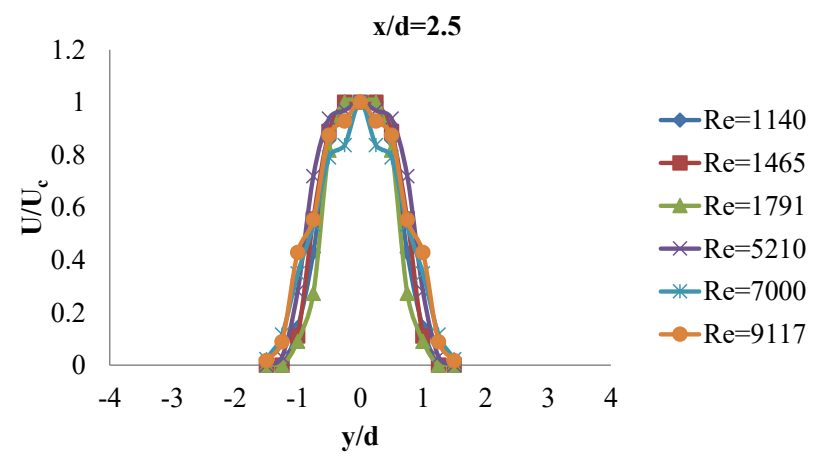

Fig. 6. Streamwise mean velocity at $x / d=2.5$

Further away from the nozzle exit section, $x / d=2.5$ (Fig. 6) and $x / d=5$ (Fig. 7), all the velocity profiles demonstrate flow symmetry and independence of Reynolds number. At these points, the shape seems to be unaltered but with higher lateral spread. For instance, in Fig. 6, at $x / d=2.5$, $y / d=1.5$ while in Fig. 7, $y / d=2.5$. Although the shape of the profile is unaltered, the lateral positions of the profile is increased, this is possibly due to gradual entrainment of ambient air. The work of [1] is used to compare the present result and good agreement is obtained. It should be noted that their measurements were at $x / d=0.04$ and $\mathrm{Re}=10,000$. Fig. 8 presents velocity profile at $x / d=10$ which falls within intermediate region based on this study, the velocity spreads further in the lateral position to $y / d=3.25$ as a result of further entrainment of ambient air.

Fig. 9-11 show the normalised centerline velocity of free jet and in long pipe for Reynolds numbers 5210, 7000 and 9117. It is observed from Fig. 9-11 that the normalised centerline velocity has almost constant values for a longer distance in the pipe, while decrease in values exist in the case of free jet across the downstream $(0 \leq x / d \leq 30)$. For instance, in Fig. 9, at jet exit, both cases have normalised velocity of one while at $x / d=3$, free jet is still 1 , while velocity in the pipe has decreased to 0.94 . Also, at $x / d=21$ free jet has 0.21 , while jet in long pipe maintained a linear value of 0.94 and later decreased to 0.81 at $x / d=29$. Also noticed is a sharp decrease in free jet. This is not surprising since the ambient fluid entrain into the jet. However, it has little or no effect in the pipe measurements. These variations also respond to change in Reynolds number. Similar trend is 
observed in other Reynolds numbers (7000 and 9117) as shown in Fig. 10, 11. This clearly indicates that there are mass redistributions in the pipe rather than entrainment of ambient fluid.

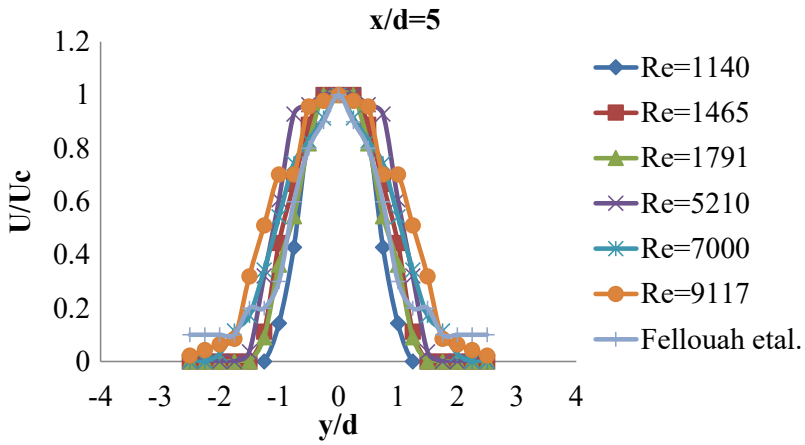

Fig. 7. Streamwise mean velocity at $x / d=5$

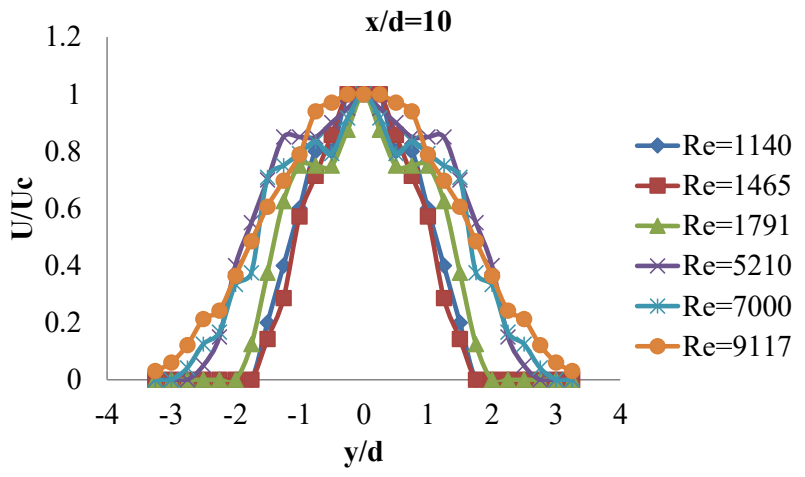

Fig. 8. Streamwise mean velocity at $x / d=10$

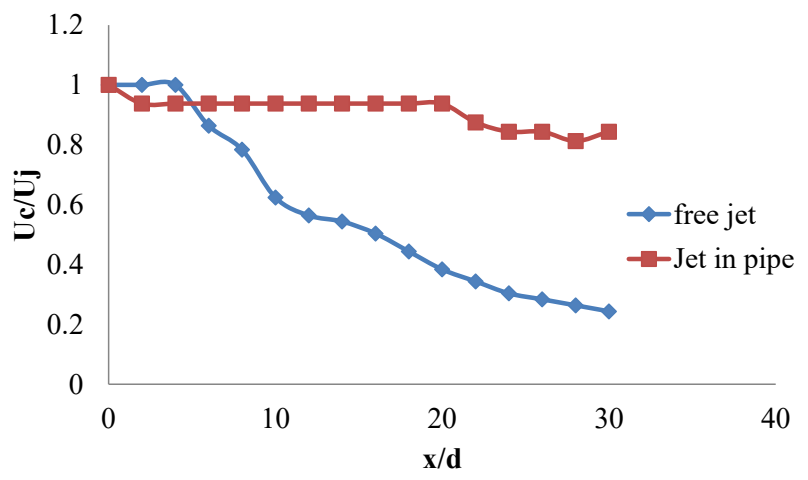

Fig. 9. Normalised mean centerline velocity for Reynolds number 5210

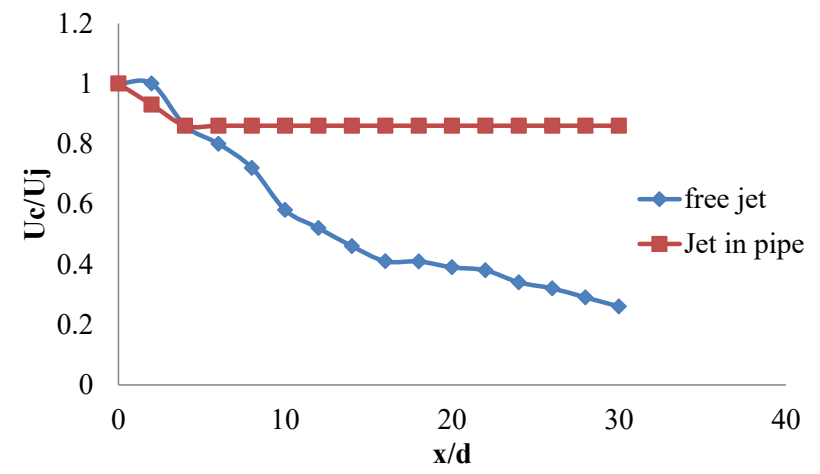

Fig. 10. Normalised mean centerline velocity for Reynolds number 7000 


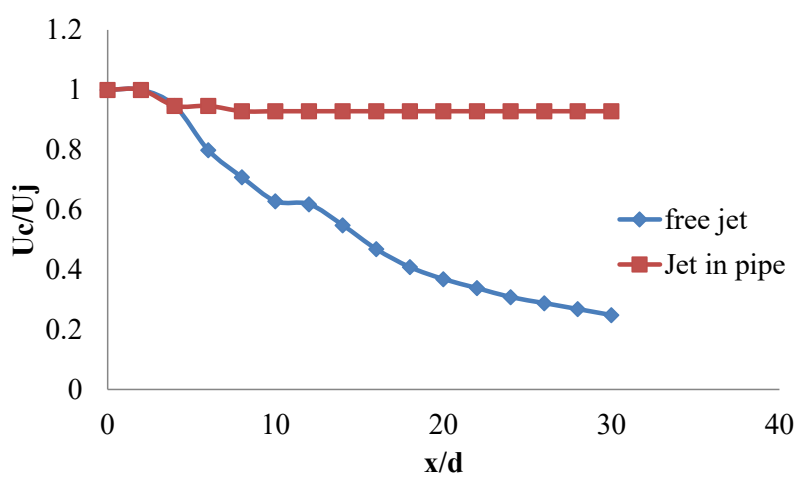

Fig. 11. Normalised mean centerline velocity for Reynolds number 9117

Overall result that stems out from all the distributions is that the Reynolds number controls the magnitude of the parameters rather than the wavelength of the oscillation.

Generally, the overall result showed that Reynolds number controlled the magnitude of the oscillation rather than the wavelength of the oscillation. Sequel to the foregoing, the objectives of this work have been achieved through experimental method which is found to be expensive. Therefore, in further work, numerical simulation is recommended to study the effect of Reynolds numbers that are beyond 9117 on round jet flow characteristics as well as other areas not captured in the scope of the present work due to exorbitant instrumentations cost. The recommended approach is hoped to further enrich our understanding of the flow development in free jet and its behavior in the attached pipe over various flow regimes. Furthermore, ambient air plays important role in the mixing of fluids in this study. Further investigation at well-controlled ambient air is required to determine the level of mixing in the shear layer. Also, potential core of $8 \mathrm{~d}$ which is outside the conventional range $(0 \leq x / d \leq 6)$ was obtained at $R e=1140$. Further investigation at lower Reynolds numbers to substantiate this is required.

\section{Conclusions}

Reynolds number effects on the development of round jet flow were studied in the region $0 \leq x / d \leq 50$ using hot-wire anemometry. Mean centerline velocity of free jet, spread of mean velocity of free jet, velocity profiles of free jet, and centerline velocity of the confined jet were measured at the flow Reynolds number of 1140, 1465, 1791, 5210, 7000 and 9117. The following conclusions were drawn:

1. In the mean centerline velocity of free jet, measurements revealed that Reynolds number dictate the potential core length such that the higher the Reynolds number, the lower the potential core which is a measure of mixing of jet and ambient fluid. However, near field could extend beyond $x / d=6$ depending on applied Reynolds number, although further investigation still required at very low Reynolds numbers.

2. Spread of the free jet as measured by jet-half width revealed that the higher the Reynolds number, the higher the spread of the mean velocity.

3. Also, velocity profiles of the free jet showed that round nozzle jet has a top-hat shape at the exit of the nozzle irrespective of the Reynolds number with higher magnitude away from the nozzle exit section.

4. In addition, centerline velocity of the confined jet revealed almost constant value of normalised velocity in axial direction when compared with free jet. This was largely due to mass redistribution rather than entrainment of ambient fluid in the pipe.

\section{References}

[1] Fellouah, H., Ball, C. G., Pollard, A. (2009). Reynolds number effects within the development region of a turbulent round free jet. International Journal of Heat and Mass Transfer, 52 (17-18), 3943-3954. doi: https://doi.org/10.1016/ j.ijheatmasstransfer.2009.03.029

[2] Fellouah, H., Pollard, A. (2009). The velocity spectra and turbulence length scale distributions in the near to intermediate regions of a round free turbulent jet. Physics of Fluids, 21 (11), 115101. doi: https://doi.org/10.1063/1.3258837 
[3] Abdel-Rahman, A. (2005). A Review of Effects of Initial and Boundary Conditions on Turbulent Jets. WSEAS transactions on fluid mechanics, 5 (4), 257-275. Available at: http://www.wseas.us/e-library/transactions/fluid/2010/52-334.pdf

[4] Dewan, A., Pathak, M., Dass, A. K. (2005). A survey of selected literature on important flow properties and computational fluid dynamics treatments of incompressible turbulent plane and round jets in quiescent ambient. Indian Journal of Engineering \& Materials Sciences, 13, 180-194. Available at: https://web.iitd.ac.in/ adewan/Dewan_2005_Review_Jets_IJEMS.pdf

[5] Ball, C. G., Fellouah, H., Pollard, A. (2012). The flow field in turbulent round free jets. Progress in Aerospace Sciences, 50, 1-26. doi: https://doi.org/10.1016/j.paerosci.2011.10.002

[6] Or, C. M., Lam, K. M., Liu, P. (2011). Potential core lengths of round jets in stagnant and moving environments. Journal of Hydro-Environment Research, 5 (2), 81-91. doi: https://doi.org/10.1016/j.jher.2011.01.002

[7] Sivakumar, S., Sangras, R., Raghavan, V. (2012). Characteristics of turbulent round jets in its potential-core region. World Academy of Science, Engineering and Technology, 61, 526-532. Available at: http://citeseerx.ist.psu.edu/viewdoc/ summary?doi=10.1.1.309.9595

[8] Xia, L. P., Lam, K. M. (2009). Velocity and concentration measurements in initial region of submerged round jets in stagnant environment and in coflow. Journal of Hydro-Environment Research, 3 (1), 21-34. doi: https://doi.org/10.1016/j.jher.2009.03.002

[9] Burattini, P., Antonia, R. A., Rajagopalan, S. (2004). Effect of initial conditions on the far field of a Round jet. 15th Australasian Fluid Mechanics Conference. The University of Sydney. Available at: https://people.eng.unimelb.edu.au/imarusic/ proceedings/15/AFMC00156.pdf

[10] Duffet, J. C., Benaïssa, A. (2013). Influence of initial conditions on the evolution towards similarity of passive scalar in turbulent round jets. Experimental Thermal and Fluid Science, 44, 834-843. doi: https://oi.org/10.1016/j.expthermflusci.2012.09.029

[11] Capone, A., Romano, G. P. (2011). Experimental investigations on secondary structures in a fully developed turbulent jet. Journal of Physics: Conference Series, 318 (3), 032045. doi: https://doi.org/10.1088/1742-6596/318/3/032045

[12] Vouros, A. P., Panidis, T. (2013). Turbulent properties of a low Reynolds number, axisymmetric, pipe jet. Experimental Thermal and Fluid Science, 44, 42-50. doi: https://doi.org/10.1016/j.expthermflusci.2012.05.012

[13] Antoine, Y., Lemoine, F., Lebouché, M. (2001). Turbulent transport of a passive scalar in a round jet discharging into a co-flowing stream. European Journal of Mechanics - B/Fluids, 20 (2), 275-301. doi: https://doi.org/10.1016/s0997-7546(00)01120-1

[14] Xu, G., Antonia, R. (2002). Effect of different initial conditions on a turbulent round free jet. Experiments in Fluids, 33 (5), 677-683. doi: https://doi.org/10.1007/s00348-002-0523-7

[15] Ferdman, E., Otugen, M. V., Kim, S. (2000). Effect of Initial Velocity Profile on the Development of Round Jets. Journal of Propulsion and Power, 16 (4), 676-686. doi: https://doi.org/10.2514/2.5627

How to cite: Oyewola, O. M., Okediji, A., Ajide, O. O., Adaramola, M. S. (2021). Examination of Reynolds number effect on the development of round jet flow. EUREKA: Physics and Engineering, 6, 39-47. doi: https://doi.org/10.21303/24614262.2021 .001872 